\title{
Identification of Tumor Mutation Burden and Immune Infiltrates in Hepatocellular Carcinoma Based on Multi-Omics Analysis
}

\section{Lu Yin ( $\nabla$ yinlu@zju.edu.cn )}

Affiliated Hangzhou First People's Hospital, Zhejiang University School of Medicine https://orcid.org/0000-0001-7607-3351

Liuzhi Zhou

Department of Hepatobiliary and Pancreatic Surgery,The Second Affiliated Hospital,Zhejiang University School of Medicine

Rujun Xu

Department of Pathology, Affiliated Hangzhou First People's Hospital,Zhejiang University School of Medicine

\section{Research}

Keywords: Hepatocellular carcinoma, immunotherapy, tumor mutation burden, biomarker, infiltration

Posted Date: August 20th, 2020

DOI: https://doi.org/10.21203/rs.3.rs-63032/v1

License: () (i) This work is licensed under a Creative Commons Attribution 4.0 International License. Read Full License 


\section{Abstract}

Background: We aimed to explore the tumor mutational burden (TMB) and immune infiltration in HCC and investigate new biomarkers for immunotherapy.

Methods: Transcriptome and gene mutation data were downloaded from the GDC portal, including 374 HCC samples and 50 matched samples. Furthermore, we divided the samples into high and low TMB groups, and analyzed the differential genes between them with GO, KEGG, and GSEA. Cibersort was used to assess the immune cell infiltration in the samples. Finally, univariate and multivariate Cox regression analyses were performed to identify differential genes related to TMB and immune infiltration, and a risk prediction model was constructed.

Results: We found 10 frequently mutated genes, including TP53, TTN, CTNNB1, MUC16, ALB, PCLO, MUC, APOB, RYR2, and ABCA. Pathway analysis indicated that these TMB-related differential genes were mainly enriched in PI3K-AKT. Cibersort analysis showed that memory $B$ cells $(P=0.02), C D 8+T$ cells $(P=0.09)$, CD4+ memory activated T cells $(P=0.07)$, and neutrophils $(P=0.06)$ demonstrated a difference in immune infiltration between high and low TMB groups. On multivariate analysis, GABRA3 $(P=0.05)$, CECR7 $(P<0.001)$, TRIM16 $(P=0.003)$, and IL7R $(P=0.04)$ were associated with TMB and immune infiltration. The risk prediction model had an area under the curve (AUC) of 0.69 , suggesting that patients with low risk had better survival outcomes.

Conclusions: Our study demonstrated for the first time that CECR7, GABRA3, IL7R, and TRIM16L mutations were associated with TMB and promoted antitumor immunity in HCC

\section{Introduction}

Hepatocellular carcinoma (HCC) is the seventh most common cancer worldwide and the fourth leading cause of cancer-related deaths [1]. Despite the efforts made by the developing countries in the past few years, $80 \%$ of HCC cases are from these countries [1]. HCC is the main type of primary liver cancer, accounting for more than $80 \%$ of the cases [2]. In America, the incidence of HCC increased in each consecutive birth cohort through 1959, predominantly in Asian Americans. The incidence of HCC dropped significantly from the 1960 to 1969 birth cohorts, and it was expected that the incidence would drop further from 2013 to 2020, with about 1.59\% decline in men and 2.20\% decline in women. However, the epidemiological data based on the population cancer register show that the incidence of HCC has increased 4-fold in the past 40 years, and this increase in the incidence will continue in the future [3, 4, 5]. The common treatment methods for HCC include surgery, radiotherapy, chemotherapy, and immunotherapy. Despite the various treatment methods, the overall survival rate of HCC is low and the mortality rate is high. The 10-year survival rate is approximately $10 \%$, and it is accompanied by high recurrence and metastasis [6].

In recent years, immunotherapy has become a common treatment for metastatic and aggressive tumors. Tumor immunotherapy principally refers to a treatment that uses the body's own immune system to attack the cancer cells. Immunotherapy mainly covers tumor vaccines, biological therapy, CAR-T cells, and immune checkpoint inhibitors (PD-1, CTLA-4) [7]. Kim et al. reported that inhibiting PD-1/PD-L1 increased the infiltration of CD8 + T cells resulting in the killing of the tumor cells [8]. PD-1 checkpoint inhibitors such as atezolizumab and nivolumab have been shown to significantly improve the clinical symptoms of patients and prolong survival time in non-small cell lung cancer [9], renal cell carcinoma [10], breast cancer [11], and melanoma [12]. However, Chiu's study demonstrated that upregulation of PVRL1 inhibited the cytotoxic T cell response through the T-cell immunoglobulin and ITIM domain (TIGIT), thus mediating resistance to PD1 inhibitors in HCC [13]. Immunotherapy is only effective in about one-fifth of the patients, and most patients are unable to benefit from it.

Tumor mutation burden (TMB) is a new biomarker that is used to predict the effect of immunotherapy. This refers to the total number of mutations per megabase in the genome. Generally speaking, the higher the TMB, the greater the difference in the tumor tissue, and the higher the patients benefit from immunotherapy [14]. Previous studies have indicated that a high TMB predicts a better prognosis in melanoma and non-small cell lung cancer [15, 16]. However, research on TMB and immune infiltration in hepatocellular carcinoma remains inconclusive.

In this study, we collected somatic mutation data and transcriptome data from The Cancer Genome Atlas (TCGA) database. We aimed to explore the TMB combined with immune infiltrates in HCC. Our study demonstrated that CECR7, GABRA3, IL TR, and TRIM16L mutations were associated with TMB and promoted antitumor immunity in HCC.

\section{Materials And Methods}

\subsection{Data acquisition and processing}

Transcriptome data, somatic mutation data, and clinical information of $374 \mathrm{HCC}$ samples and 50 matched normal or adjacent tissue samples were obtained from the GDC portal (https://portal.gdc.cancer.gov/). In addition, the clinical data of HCC patients ( $n=376)$ comprising age, sex, survival time, survival status, grade, stage, and the American Joint Committee on Cancer (AJCC-TNM) staging were collected (Table 1).

\subsection{Classification of clinical data based on the tumor mutation burden}

TMB was defined as the total number of mutations per megabase of the genome. We processed the TMB and raw transcriptome data obtained from the GDC portal using the R software (https://www.r-project.org/) and Excel "vlookup" to obtain the TMB scores of each sample. According to the median of the TMB scores (median=2.45), we divided the HCC samples into high and low TMB groups. In addition, we analyzed the relationship between TMB scores and clinical characteristics (Figure S1).

\subsection{Multi-omics analysis}

The somatic mutation data processed by Varsan were downloaded from TCGA, and the "maftools" package (http://bioconductor.org/) was used to visualize the TMB information. Based on the Java working environment, strawberry-perl (https://www.perl.org/) was used to run the perl script to extract the genome 
mutation information of the 364 HCC patients.

Using the R language, we merged the mutation information of HCC patients with the clinical survival data. According to the median value of the TMB scores, samples were classified into high and low TMB groups. Gene ontology (GO) and gene set enrichment analysis (GSEA) were performed as previously described [17]. A p-value $<0.05$ and $\| \log$ (fold change) $>1$ | were regarded to denote enrichment significance. Cibersort is a deconvolution algorithm used to evaluate the relative abundance of immune infiltration in clinical samples. A threshold value of $\mathrm{P}<0.05$ and 1,000 repeated calculations were considered as successful.

Uni- and multivariate Cox regression analyses were conducted to assess the immune-related genes. Based on the results of the multivariate regression analysis, we constructed a risk prediction model and divided the patients into high-risk and low-risk groups according to the risk scores (TMBRS). A receiver operating characteristic (ROC) curve was used to test the sensitivity and specificity of this model. Finally, an analysis of immune cell infiltration with survival outcomes was conducted using the Kaplan-Meier method.

\subsection{Statistical analysis}

All statistical analyses were performed using R, including the Kaplan-Meier survival analysis and uni- and multivariate Cox regression analyses. For all comparisons, $\mathrm{P}<0.05$ was considered to indicate statistical significance.

\section{Results}

\subsection{Landscape of frequent gene mutations in hepatocellular carcinoma}

We obtained the mutation data of $364 \mathrm{HCC}$ samples processed by "Varsan" from the GDC portal and the "maftools" package was used to visualize the data. Among somatic mutations, missense mutations were the most common type (Figure 1a), single nucleotide polymorphisms had more components than insertions or deletions (Figure 1b), and C>T mutations were the most common (Figure 1c). The tumor mutation burden of each sample was shown using various colors in box plots (Figure 1d, 1e). A waterfall plot displays the comprehensive mutation data of each sample. In addition, we selected the 10 most frequently mutated genes in the American HCC samples obtained from the TCGA cohort, including TP53 (30\%), TTN (24\%), CTNNB1 (25\%), MUC16 (14\%), ALB (13\%), PCLO (10\%), MUC (9\%), APOB (9\%), RYR2 (9\%), and ABCA (8\%) (Figure 1f, 1g). Furthermore, we performed co-expression analysis of these mutant genes (Figure 1h).

\subsection{Gene Ontology and KEGG Analysis of Gene Expression in the Two TMB Groups}

Differentially expressed genes were obtained through R software (Figure S2). The "Limma" package was used to analyze the differentially expressed genes in the two TMB groups, and 375 differentially expressed genes were obtained. Differential genes were identified by log (fold change) $>1$ and $P<0.05$. For the biological processes, we used Gene Ontology to enrich the differential genes, including biological process (BP), cellular component (CC), and molecular function (MF). With regard to BP, these differential genes were mainly enriched in the extracellular matrix organization. With regard to $\mathrm{CC}$ and MF, the differential genes were predominantly enriched in the collagen-containing extracellular matrix and matrix structural constituent, respectively (Figure $2 \mathrm{a}$, $2 \mathrm{~b}$ ). Furthermore, we used KEGG to conduct pathway analysis on these differentially expressed genes, and found that the TMB-related differential genes were mainly enriched in the PI3K-Akt signaling pathway, a pathway that has been clearly reported to be related to cancer. In addition, the KEGG analysis revealed that they were also significantly enriched in cytokine-cytokine receptor interaction and focal adhesion pathway (Figure 2c, 2d, Table 2).

\subsection{Gene Set Enrichment Analysis(GSEA) with high and low TMB groups}

GSEA was used to analyze the TCGA cohort. We found that in the high TMB group, the differentially expressed genes were mainly enriched in the proteasome, drug metabolism other enzymes, porphyrin, and chlorophyll metabolism (Figure 3a-3c). In the low TMB group, the differentially expressed genes chiefly participated in ECM receptor interaction, vascular smooth muscle contraction, and ether lipid metabolism (Figure 3d-3f). The top 10 differentially expressed GSEA outcomes in the high and low TMB groups are shown in Tables S1 and S2.

\subsection{Tumor-infiltrating immune cells in hepatocellular carcinoma}

Based on the Cibersort algorithm, we analyzed the infiltration of 22 immune cells in the HCC microenvironment. As shown in Figure 4a, each column represents a sample, and different colors represent different immune cells. It was discovered that the 22 kinds of tumor-infiltrating immune cells varied significantly in each sample. Furthermore, we used the R software to process them by dividing them into high and low TMB groups, and compared the immune cell infiltration between the two groups. It was found that the memory $B$ cells $(P=0.027)$ demonstrated a significantly differential expression in the high and low TMB groups. CD8+ T cells $(P=0.09)$, CD4+ memory activated T cells $(P=0.07)$ and neutrophils $(P=0.06)$ also showed differences in expression between the high and low TMB groups (Figure 4b).

\subsection{Identification of significant immune genes for HCC prognostication}

Immune-related genes were downloaded from the database (http://www.immport.org/) and intersected with the differential genes in the sample to obtain 51 related differential immune genes (Figure 5a). Subsequently, we performed univariate Cox regression analysis using these differential immune genes, and selected genes with statistical significance for prognosis, including GABRA3, LUCAT1, MAGEA12, CECR7, STEAP4, CSAG1, TRIM16L, LINC00958, IL 7R, and MAGEA3 (Table 3). Multivariate Cox regression analysis was performed as described above and the differential immune genes obtained were $G A B R A 3$, $C E C R 7, T R I M 16 L$, and ILTR. In addition, we performed survival analysis (Kaplan-Meier method) on these four immune genes and found that the mutations in GABRA3, CECR7, TRIM16, and IL7R were related to TMB and promoted anti-tumor immunity. The low expression of GABRA3, CECR7, and TRIM1,6 and the 
high expression of IL7R significantly prolonged the survival time of patients (Figure $5 \mathrm{~b}-5 \mathrm{e}$, Table 4). Furthermore, the associations between $G A B R A 3, C E C R 7$, TRIM16, and IL7R mutations, and immune cell infiltration are shown in Figure S3.

\subsection{Analysis and evaluation of the risk prediction model}

Based on the multivariate Cox regression analysis, we constructed a risk prediction model and the coefficients are shown in Table 4. We divided the TCGA cohort into a high-risk group and low-risk group according to the median of the risk scores (median=0.96), and then performed Kaplan-Meier analysis. It was found that the survival rate of the high-risk group was significantly reduced ( $P=0.002$, Figure $6 \mathrm{a})$. Additionally, by analyzing the ROC curve of the model, we found that the established model had certain clinical significance for the prognosis of HCC (area under the curve [AUC] $=0.691$, Figure $6 \mathrm{~b}$ ).

\section{Discussion}

$\mathrm{HCC}$ is the fourth leading cause of cancer-related deaths worldwide, with approximately 750,000 new cases each year [1, 18]. Once HCC is diagnosed, it is often an aggressive or metastatic cancer, and $70 \%$ of the patients receive palliative treatment $[19,20]$. The development of HCC is a complex process involving more than 160 genetic changes [21]. In recent years, immunotherapy has achieved great success in the treatment of HCC. Immunotherapy is the use of drugs to promote immune cell infiltration in the tumor microenvironment leading to the killing of the tumor cells by T or B lymphocytes. The most common first-line treatment for HCC is sorafenib, which has been proven to prolong the survival of patients with HCC for several months [22, 23]. Immunotherapy mainly includes tumor vaccines, biological therapy, CAR-T cells, and immune checkpoint inhibitors (PD-1, CTLA-7). Liu et al. reported that the BET protein inhibitor JQ1 can enhance the expression of Rab8A and further upregulate the expression of PD-1, which can enhance the tumor-killing effect of CD8 + cells [24]. In addition to the immune checkpoint inhibitors, tumor vaccines have also been widely used in the treatment of HCC. Zhen et al. indicated that exosomes derived from dendritic cells stimulated the tumor immune response in HCC and killed or inhibited the tumor cells [25]. Tumor immunotherapy has been widely reported to achieve good results in lung cancer, kidney cancer, and melanoma [26].

Although immunotherapy has achieved great success, only a small proportion of patients benefit from it. Previous studies have revealed that patients with a high TMB had accompanying obvious immune cell infiltration and often benefited from immunotherapy. Therefore, there is an urgent need for biomarkers to predict the effect of immunotherapy. Zhu et al. found that the EP300 mutation was related to the TMB and promoted anti-tumor immunity in bladder cancer [27]. Zhang et al. reported that immune genes such as CES1 and CSAG1 could be used as biomarkers for predicting the effects of immunotherapy through a multi-omics analysis [28]. Similarly, Pan et al. discovered that Laylin is a prognostic marker of survival and is associated with immune infiltration in gastric and colon cancer [29]. However, the TMB and immune infiltration remain unclear in HCC.

In this study, we downloaded a dataset of 364 American HCC samples from the TCGA. By comparing the immune-infiltrating cells in the high and low TMB groups through the violin chart, we found that memory B cells, CD $8+T$ cells, and CD 4 + memory activated T cells were more abundant in the high TMB group. Gibert's study reported that human memory B cells produced anti-IgG antibodies to kill tumor cells in melanoma [30]. In HCC, previous studies have reported that the immune infiltration of CD8 $+\mathrm{T}$ cells and CD $4+$ memory activated $\mathrm{T}$ cells was associated with better clinical results and prognosis [8, 31].

Differential genes were selected by both GO analysis and KEGG analysis, and then GSEA analysis was performed on the differential genes of the high and low TMB groups. Four related differential genes were identified through univariate and multivariate cox regression analyses, including GABRA3, CECR7, TRIM16L, and $I L 7 R$. Furthermore, we constructed a risk prediction model. The AUC value of the ROC curve was 0.69 , which implied that our model had a certain accuracy. Patients with high-risk values benefited from immunotherapy and had a better prognosis. As far as we know, this was the first study to combine TMB with immune infiltration to construct a risk prediction model in HCC. However, additional samples need to be analyzed before clinical application, and we need to consider further optimizing the ROC curve with other prognostic biomarkers to increase the AUC value.

The main limitation of this study was that we only included samples from the TCGA database and lacked clinical validation experiments. Even if $G A B R A 3$, CECR7, TRIM16L, and IL TR are frequently mutated in HCC in the United States and are associated with TMB and immune infiltration, this does not mean that this risk prediction model can provide a basis for immunotherapy in actual clinical practice. Further clinical trials are needed for verifying this risk prediction model.

In conclusion, our study proved that GABRA3, CECR7, TRIM16L, and IL 7R were frequently mutated in HCC and related to TMB and immune infiltration. In addition, we built a prognostic model to evaluate the effect of immunotherapy in HCC. These results suggest novel biomarkers for immunotherapy in HCC.

\section{Conclusions}

Our study demonstrated for the first time that CECR7, GABRA3, IL7R, and TRIM16L mutations were associated with TMB and promoted antitumor immunity in HCC.

\section{Abbreviations}

$\mathrm{HCC}$

hepatocellular carcinoma; TMB:tumor mutation burden; TCGA:The Cancer Genome Atlas; GO:Gene Ontology; GSEA:Gene set enrichment analysis; TNM:tumornode-metastasis; HR:hazard ratio.

\section{Declarations}


Not applicable.

\section{Author's contribution}

L.Y. and L.Z. conceived and designed the study and analyzed the data. L.Z. drafted the article. R.X. reviewed the manuscript. All authors read and approved the manuscript.

\section{Funding}

There are no funders to report for this submission.

\section{Availability of data and materials}

The dataset used and/or analyzed during the current study are available from the corresponding author on reasonable request.

\section{Consent for publication}

All authors agree to submit the article for publication.

\section{Competing interests}

The authors declare that they have no competing interests.

\section{Author details}

1 Department of Pathology, Affiliated Hangzhou First People's Hospital, Zhejiang University School of Medicine, Hangzhou 310006, China.

2 Department of Hepatobiliary and Pancreatic Surgery, The Second Affiliated Hospital, Zhejiang University School of Medicine, Hangzhou 310009, China

\section{References}

1. Yang, J. D., Hainaut, P., Gores, G. J., Amadou, A., Plymoth, A., \& Roberts, L. R. A global view of hepatocellular carcinoma: trends, risk, prevention and management. Nature reviews. Gastroenterology \& hepatology. 2019;16(10): 589-604.

2. El-Serag, H. B., \& Rudolph, K. L. (2007). Hepatocellular carcinoma: epidemiology and molecular carcinogenesis. Gastroenterology. 2007;132(7):25572576.

3. Petrick, J. L., Kelly, S. P., Altekruse, S. F., McGlynn, K. A., \& Rosenberg, P. S. Future of Hepatocellular Carcinoma Incidence in the United States Forecast Through 2030. Journal of clinical oncology: official journal of the American Society of Clinical Oncology. 2016; 34(15): 1787-1794.

4. Massarweh NN, El-Serag HB. Epidemiology of Hepatocellular Carcinoma and Intrahepatic Cholangiocarcinoma. Cancer Control. 2017;24(3):1073274817729245. doi:10.1177/1073274817729245

5. Singal, A. G., Lampertico, P., \& Nahon, P. Epidemiology and surveillance for hepatocellular carcinoma: New trends. Journal of hepatology. 2020;72(2): 250-261.

6. Siegel, R. L., Miller, K. D., \& Jemal, A. CA: a cancer journal for clinicians. Cancer statistics. 2016; 66(1): 7-30.

7. Prieto, J., Melero, I., \& Sangro, B. Immunological landscape and immunotherapy of hepatocellular carcinoma. Nature reviews. Gastroenterology \& hepatology. 2015;12(12): 681-700.

8. Kim, H. D., Song, G. W., Park, S., Jung, M. K., Kim, M. H., Kang, H. J., Yoo, C., Yi, K., Kim, K. H., Eo, S., Moon, D. B., Hong, S. M., Ju, Y. S., Shin, E. C., Hwang, S., \& Park, S. H. Association Between Expression Level of PD1 by Tumor-Infiltrating CD8+ T Cells and Features of Hepatocellular Carcinoma.

Gastroenterology.2018; 155(6): 1936-1950.e17.

9. Chardin, D., Paquet, M., Schiappa, R., Darcourt, J., Bailleux, C., Poudenx, M., Sciazza, A., Ilie, M., Benzaquen, J., Martin, N., Otto, J., \& Humbert, O. Baseline metabolic tumor volume as a strong predictive and prognostic biomarker in patients with non-small cell lung cancer treated with PD1 inhibitors: a prospective study. Journal for immunotherapy of cancer.2020; 8(2): e000645.

10. Buchbinder, E. I., Dutcher, J. P., Daniels, G. A., Curti, B. D., Patel, S. P., Holtan, S. G., Miletello, G. P., Fishman, M. N., Gonzalez, R., Clark, J. I., Richart, J. M., Lao, C. D., Tykodi, S. S., Silk, A. W., \& McDermott, D. F. Therapy with high-dose Interleukin-2 (HD IL-2) in metastatic melanoma and renal cell carcinoma following PD1 or PDL1 inhibition. Journal for immunotherapy of cancer.2019; 7(1): 49.

11. Szekely, B., Bossuyt, V., Li, X., Wali, V. B., Patwardhan, G. A., Frederick, C., Silber, A., Park, T., Harigopal, M., Pelekanou, V., Zhang, M., Yan, Q., Rimm, D. L., Bianchini, G., Hatzis, C., \& Pusztai, L. Immunological differences between primary and metastatic breast cancer. Annals of oncology: official journal of the European Society for Medical Oncology.2018; 29(11): 2232-2239.

12. Luke, J. J., Flaherty, K. T., Ribas, A., \& Long, G. V. Targeted agents and immunotherapies: optimizing outcomes in melanoma. Nature reviews. Clinical oncology.2017; 14(8): 463-482.

13. Chiu, D. K., Yuen, V. W., Wing-Sum Cheu, J., Wei, L. L., Ting, V., Fehlings, M., Sumatoh, H., Nardin, A., Newell, E. W., Oi-Lin Ng, I., Yau, T. C., Wong, C. M., \& Wong, C. C. Hepatocellular Carcinoma Cells Up-regulate PVRL1, Stabilizing Poliovirus Receptor and Inhibiting the Cytotoxic T-Cell Response via TIGIT to Mediate Tumor Resistance to PD1 Inhibitors in Mice. Gastroenterology. 2020; S0016-5085(20)30461-3. Advance online publication. 
14. Chan, T. A., Yarchoan, M., Jaffee, E., Swanton, C., Quezada, S. A., Stenzinger, A., \& Peters, S. Development of tumor mutation burden as an immunotherapy biomarker: utility for the oncology clinic. Annals of oncology: official journal of the European Society for Medical Oncology. 2019; 30(1):44-56.

15. Chen, H., Chong, W., Wu, Q., Yao, Y., Mao, M., \& Wang, X. Association of LRP1B Mutation With Tumor Mutation Burden and Outcomes in Melanoma and Non-small Cell Lung Cancer Patients Treated With Immune Check-Point Blockades. Frontiers in immunology.2019; $10: 1113$.

16. Chen, Y., Liu, Q., Chen, Z., Wang, Y., Yang, W., Hu, Y., Han, W., Zeng, H., Ma, H., Dai, J., \& Zhang, H. PD-L1 expression and tumor mutational burden status for prediction of response to chemotherapy and targeted therapy in non-small cell lung cancer. Journal of experimental \& clinical cancer research : CR. 2019; 38(1): 193.

17. Subramanian, A., Tamayo, P., Mootha, V. K., Mukherjee, S., Ebert, B. L., Gillette, M. A., Paulovich, A., Pomeroy, S. L., Golub, T. R., Lander, E. S., \& Mesirov, J. P. Gene set enrichment analysis: a knowledge-based approach for interpreting genome-wide expression profiles. Proceedings of the National Academy of Sciences of the United States of America. 2015; 102(43): 15545-15550.

18. Ferlay, J., Soerjomataram, I., Dikshit, R., Eser, S., Mathers, C., Rebelo, M., Parkin, D. M., Forman, D., \& Bray, F. Cancer incidence and mortality worldwide: sources, methods and major patterns in GLOBOCAN 2012. International journal of cancer.2015;136(5):E359-E386.

19. Vogel, A., Cervantes, A., Chau, I., Daniele, B., Llovet, J. M., Meyer, T., Nault, J. C., Neumann, U., Ricke, J., Sangro, B., Schirmacher, P., Verslype, C., Zech, C. J., Arnold, D., \& Martinelli, E. Hepatocellular carcinoma: ESMO Clinical Practice Guidelines for diagnosis, treatment and follow-up. Annals of oncology: official journal of the European Society for Medical Oncology.2019; 30(5): 871-873.

20. Llovet, J. M., Zucman-Rossi, J., Pikarsky, E., Sangro, B., Schwartz, M., Sherman, M., \& Gores, G. Hepatocellular carcinoma. Nature reviews. Disease primers.2016; 2: 16018

21. Schulze, K., Imbeaud, S., Letouzé, E., Alexandrov, L. B., Calderaro, J., Rebouissou, S., Couchy, G., Meiller, C., Shinde, J., Soysouvanh, F., Calatayud, A. L., Pinyol, R., Pelletier, L., Balabaud, C., Laurent, A., Blanc, J. F., Mazzaferro, V., Calvo, F., Villanueva, A., Nault, J. C., ... Zucman-Rossi, J. Exome sequencing of hepatocellular carcinomas identifies new mutational signatures and potential therapeutic targets. Nature genetics. 2015; 47(5): 505-511.

22. Kim, D. W., Talati, C., \& Kim, R. Hepatocellular carcinoma (HCC): beyond sorafenib-chemotherapy. Journal of gastrointestinal oncology.2017; 8(2): 256265.

23. Flynn, M. J., Sayed, A. A., Sharma, R., Siddique, A., \& Pinato, D. J. Challenges and Opportunities in the Clinical Development of Immune Checkpoint Inhibitors for Hepatocellular Carcinoma. Hepatology (Baltimore, Md.).2019; 69(5): 2258-2270.

24. Liu, C., Miao, X., Wang, Y., Wen, L., Cheng, X., Kong, D., Zhao, P., Song, D., Wang, X., Ding, X., Xia, H., Wang, W., Sun, Q., \& Gong, W. BET Protein Inhibition Improves Immunotherapy Efficacy in Hepatocellular Carcinoma. Cancer science.2020; 10.1111/cas.14588. Advance online publication.

25. Lu, Z., Zuo, B., Jing, R., Gao, X., Rao, Q., Liu, Z., Yin, H. Dendritic cell-derived exosomes elicit tumor regression in autochthonous hepatocellular carcinoma mouse models. J Hepatol.2017; 67(4): 739-748.

26. Larkin, J., Chiarion-Sileni, V., Gonzalez, R., Grob, J. J., Cowey, C. L., Lao, C. D., Schadendorf, D., Dummer, R., Smylie, M., Rutkowski, P., Ferrucci, P. F., Hill, A., Wagstaff, J., Carlino, M. S., Haanen, J. B., Maio, M., Marquez-Rodas, I., McArthur, G. A., Ascierto, P. A., Long, G. V., ... Wolchok, J. D. Combined Nivolumab and Ipilimumab or Monotherapy in Untreated Melanoma. The New England journal of medicine.2015; 373(1): $23-34$.

27. Zhu, G., Pei, L., Li, Y., \& Gou, X. EP300 mutation is associated with tumor mutation burden and promotes antitumor immunity in bladder cancer patients. Aging.2020; 12(3): 2132-2141.

28. Zhang, C., Shen, L., Qi, F., Wang, J., \& Luo, J. Multi-omics analysis of tumor mutation burden combined with immune infiltrates in bladder urothelial carcinoma. Journal of cellular physiology.2020; 235(4): 3849-3863.

29. Pan, J. H., Zhou, H., Cooper, L., Huang, J. L., Zhu, S. B., Zhao, X. X., Ding, H., Pan, Y. L., \& Rong, L. LAYN Is a Prognostic Biomarker and Correlated With Immune Infiltrates in Gastric and Colon Cancers. Frontiers in immunology.2019; $10: 6$.

30. Gilbert, A. E., Karagiannis, P., Dodev, T., Koers, A., Lacy, K., Josephs, D. H., Takhar, P., Geh, J. L., Healy, C., Harries, M., Acland, K. M., Rudman, S. M., Beavil, R. L., Blower, P. J., Beavil, A. J., Gould, H. J., Spicer, J., Nestle, F. O., \& Karagiannis, S. N. Monitoring the systemic human memory B cell compartment of melanoma patients for anti-tumor IgG antibodies. PloS one.2011; 6(4): e19330.

31. Chew, V., Lai, L., Pan, L., Lim, C. J., Li, J., Ong, R., Chua, C., Leong, J. Y., Lim, K. H., Toh, H. C., Lee, S. Y., Chan, C. Y., Goh, B., Chung, A., Chow, P., \& Albani, S. Delineation of an immunosuppressive gradient in hepatocellular carcinoma using high-dimensional proteomic and transcriptomic analyses. Proceedings of the National Academy of Sciences of the United States of America.2017; 114(29): E5900-E5909.

\section{Tables}


Table 1

Basic clinical information of all 376

HCC patients from TCGA cohort

\begin{tabular}{|c|c|}
\hline Variables & $\begin{array}{l}\text { TCGA cohorts } \\
(n=376)\end{array}$ \\
\hline Age & $59.45 \pm 13.49$ \\
\hline Gender & $122(32.4 \%)$ \\
\hline Female & $254(67.6 \%)$ \\
\hline \multicolumn{2}{|l|}{ Male } \\
\hline Tumor Grade & $235(62.5 \%)$ \\
\hline $\mathrm{G} 1 / \mathrm{G} 2$ & $123(32.7 \%)$ \\
\hline G3 & $13(3.5 \%)$ \\
\hline G4 & $5(1.3 \%)$ \\
\hline \multicolumn{2}{|l|}{ Unknow } \\
\hline Pathologic Stage & $261(69.4 \%)$ \\
\hline I\&l| & $91(24.2 \%)$ \\
\hline III\&IV & $24(6.4 \%)$ \\
\hline \multicolumn{2}{|l|}{ Unknow } \\
\hline AJCC-T & $185(49.2 \%)$ \\
\hline T1 & $94(25.0 \%)$ \\
\hline T2 & $81(21.5 \%)$ \\
\hline T3 & $13(3.5 \%)$ \\
\hline T4 & $3(0.8 \%)$ \\
\hline \multicolumn{2}{|l|}{ Unknow } \\
\hline AJCC-N & $257(68.4 \%)$ \\
\hline NO & $4(1.1 \%)$ \\
\hline N1-N3 & $115(30.6 \%)$ \\
\hline \multicolumn{2}{|l|}{ Unknow } \\
\hline AJCC-M & $272(72.3 \%)$ \\
\hline MO & $4(1.1 \%)$ \\
\hline M1 & $100(26.6 \%)$ \\
\hline Unknow & \\
\hline
\end{tabular}




\begin{tabular}{|c|c|c|c|c|}
\hline Description & GeneRation & p.adj & q & genelD \\
\hline $\begin{array}{l}\text { Viral } \\
\text { protein } \\
\text { interaction } \\
\text { with } \\
\text { cytokine } \\
\text { and } \\
\text { cytokine } \\
\text { receptor }\end{array}$ & $9 / 115$ & 0.002 & 0.002 & CXCL6/CCL22/CCL21/CCL2/PF4V1/CCL19/PPBP/IL34/IL6 \\
\hline $\begin{array}{l}\text { ECM- } \\
\text { receptor } \\
\text { interaction }\end{array}$ & $8 / 115$ & 0.003 & 0.002 & ITGB8/LAMC2/LAMA2/COMP/THBS1/TNXB/ITGB4/THBS2 \\
\hline $\begin{array}{l}\text { PI3K-Akt } \\
\text { signaling } \\
\text { pathway }\end{array}$ & $16 / 115$ & 0.003 & 0.002 & NTRK2/ITGB8/LAMC2/LAMA2/COMP/FGF1/THBS1/IGF2/TNXB/IL6/IL7R/PDGFD/ITGB4/THBS2/TGFA/VI \\
\hline $\begin{array}{l}\text { Focal } \\
\text { adhesion }\end{array}$ & $11 / 115$ & 0.007 & 0.006 & MYL9/ITGB8/LAMC2/LAMA2/COMP/THBS1/TNXB/PDGFD/ITGB4/THBS2/VEGFD \\
\hline $\begin{array}{l}\text { Cytokine- } \\
\text { cytokine } \\
\text { receptor } \\
\text { interaction }\end{array}$ & $13 / 115$ & 0.009 & 0.009 & CXCL6/CCL22/CCL21/CCL2/PF4V1/CCL19/PPBP/EDAR/TNFSF15/LTB/IL34/IL6/IL7R \\
\hline $\begin{array}{l}\text { Bile } \\
\text { secretion }\end{array}$ & $7 / 115$ & 0.009 & 0.009 & NCEH1/SCTR/SLC5A1/CFTR/UGT1A3/AQP1/FXYD2 \\
\hline Malaria & $5 / 115$ & 0.020 & 0.018 & CCL2/COMP/THBS1/IL6/THBS2 \\
\hline
\end{tabular}

Table 3

Univariate Cox analysis of TMB related genes combined with

\begin{tabular}{|lllll|}
\hline \multicolumn{5}{c}{ immune infiltrates in HCC } \\
\hline Gene & HR & HR.95L & HR.95H & Cox P value \\
\hline LUCAT1 & 1.172311 & 1.084596 & 1.26712 & $6.16 \mathrm{E}-05$ \\
\hline MAGEA12 & 1.032631 & 1.013041 & 1.0526 & 0.001017 \\
\hline CECR7 & 1.677537 & 1.391016 & 2.023075 & $6.17 \mathrm{E}-08$ \\
\hline STEAP4 & 0.84268 & 0.714068 & 0.994456 & 0.04279 \\
\hline CSAG1 & 1.019108 & 1.006541 & 1.031832 & 0.002791 \\
\hline TRIM16L & 1.024846 & 1.010713 & 1.039177 & 0.000532 \\
\hline LINC00958 & 1.144123 & 1.020469 & 1.282759 & 0.021044 \\
\hline IL7R & 0.866068 & 0.768572 & 0.975932 & 0.018286 \\
\hline MAGEA3 & 1.021312 & 1.008233 & 1.03456 & 0.001342 \\
\hline
\end{tabular}

Table 4

multivariate cox analysis of TMB related genes combined with immune infiltrates

\begin{tabular}{|llllll|}
\hline Gene & coef & HR & HR.95L & HR.95H & pvalue \\
\hline GABRA3 & 0.13787 & 1.147827 & 0.997744 & 1.320485 & 0.053808 \\
\hline CECR7 & 0.4248 & 1.529284 & 1.245496 & 1.877735 & $4.99 \mathrm{E}-05$ \\
\hline TRIM16L & 0.021488 & 1.021721 & 1.00718 & 1.036471 & 0.0033 \\
\hline IL7R & -0.12144 & 0.885647 & 0.787232 & 0.996365 & 0.043325 \\
\hline
\end{tabular}

\section{Figures}



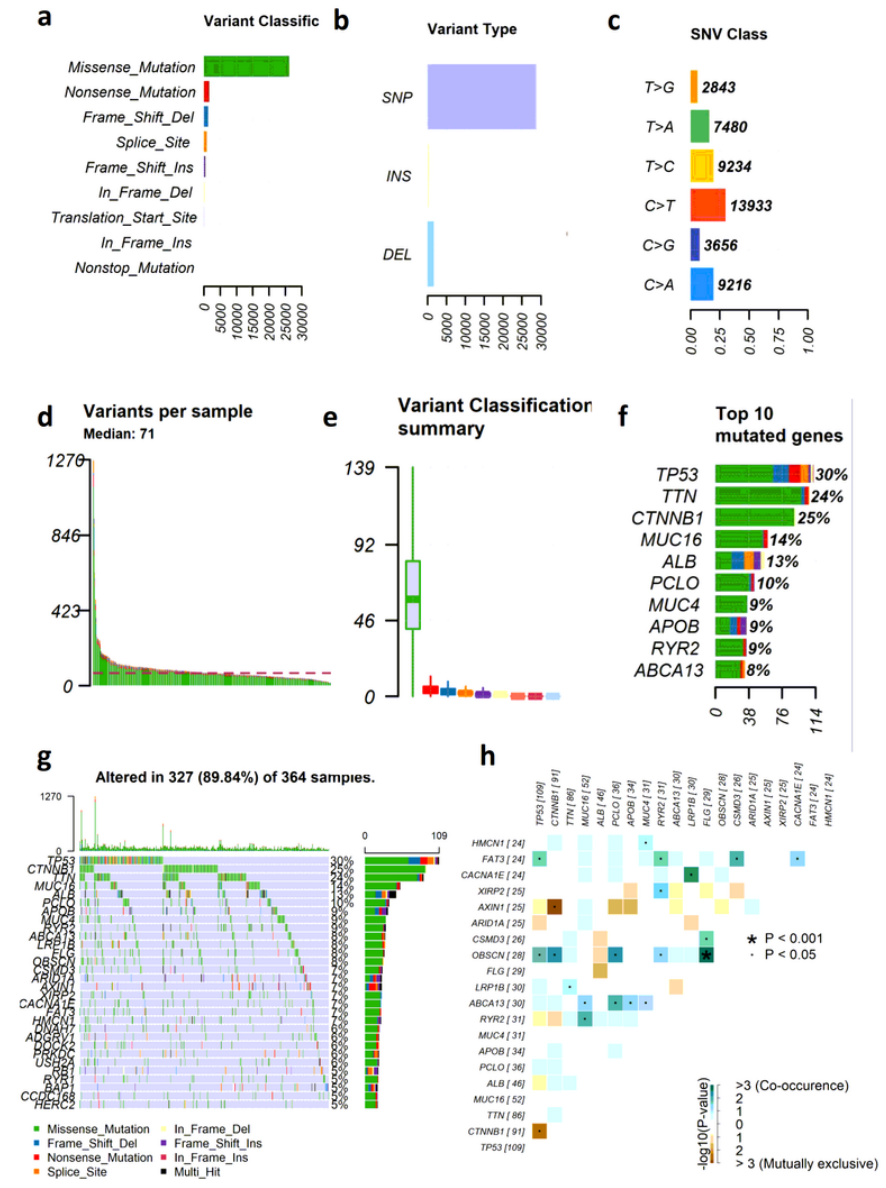

\section{Figure 1}

Landscape of frequently gene mutation in $\mathrm{HCC}(\mathrm{a}-\mathrm{c})$ Statistical calculations of mutation types based on different categories, where missense mutation, SNP, C>T mutation were the most (d-e) Display of TMB in each HCC sample (f) The top 10 mutant genes in HCC, including TP53, TTN, CTNNB1, MUC16, ALB, PCLO, MUC, APOB, RYR2, ABCA. (g) Landscape of mutation information of each HCC sample in waterfall plot. Each column represents a sample (h) Co-expression of mutant genes in HCC. HCC, hepatocellular carcinoma; SNP, single nucleotide polymorphism. ${ }^{*} \mathrm{P}<0.001,{ }^{\prime} \mathrm{P}<0.05$. 

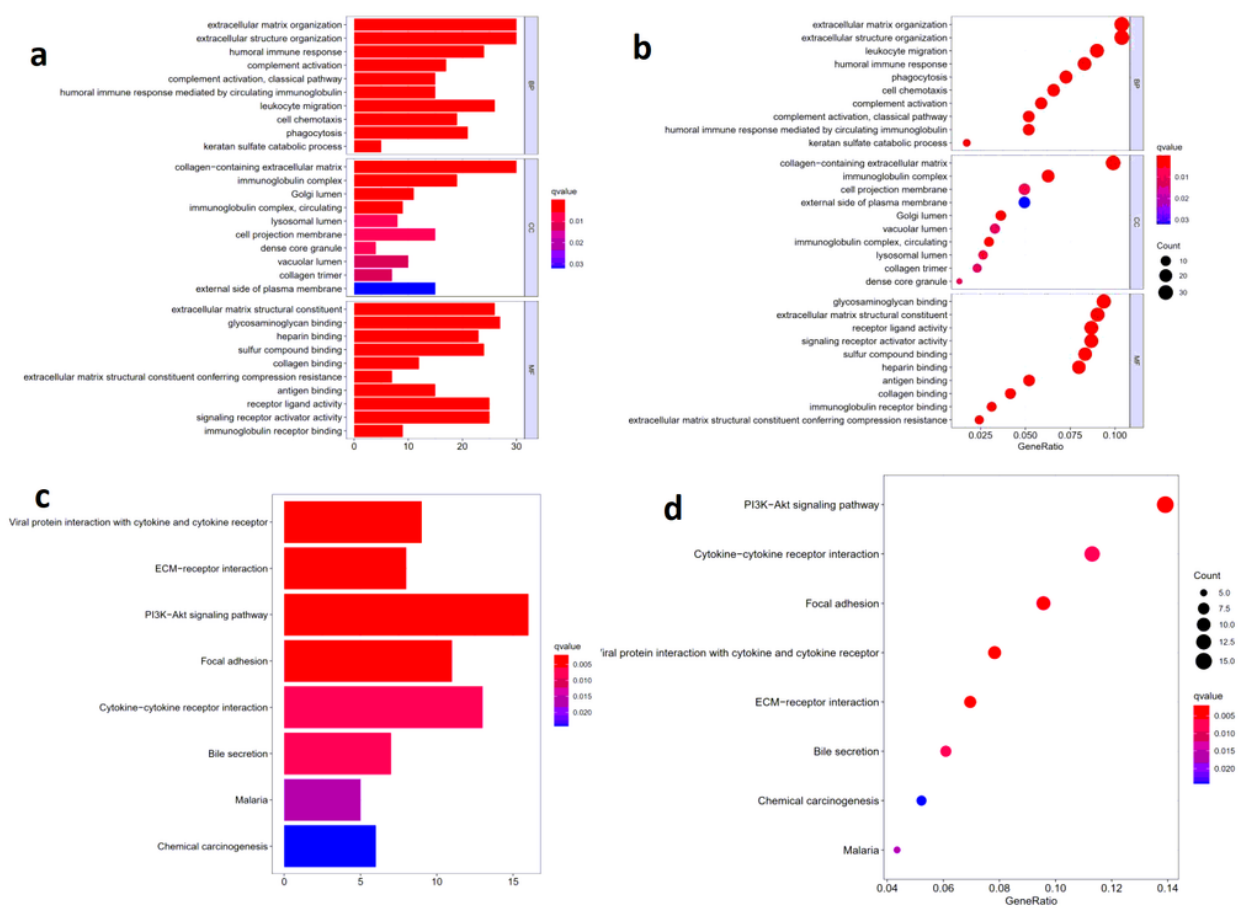

Figure 2

Gene Ontology and KEGG analysis of the gene expression of the two TMB groups (a-b) GO enriched analysis of differential genes in BP, CC, MP. (c-d) KEGG analysis with these differential genes were enriched in PI3K-AKt, cytokine-cytokine receptor interaction and focal adhesion axis. TMB: tumor mutation burden; BP: biological process; CC, cellular component; MP, molecular function; KEGG, Kyoto Encyclopedia of Genes and Genomes. 
a

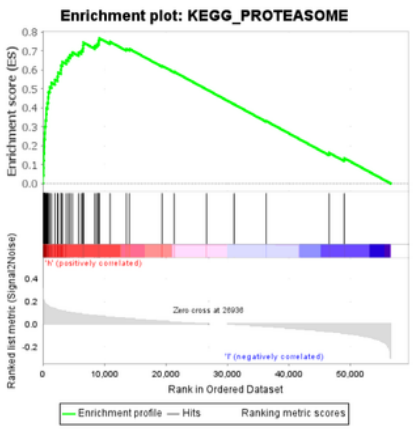

d

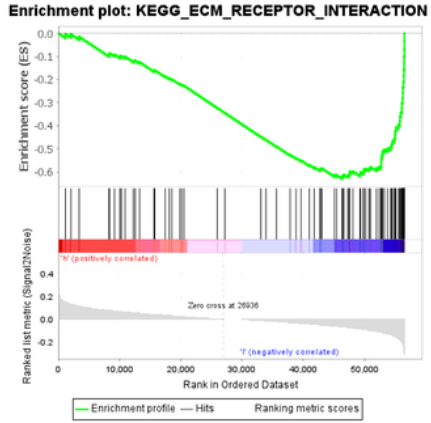

Enrichment plot:

b KEG__DRUG_METABOLISM_OTHER_ENZYMES
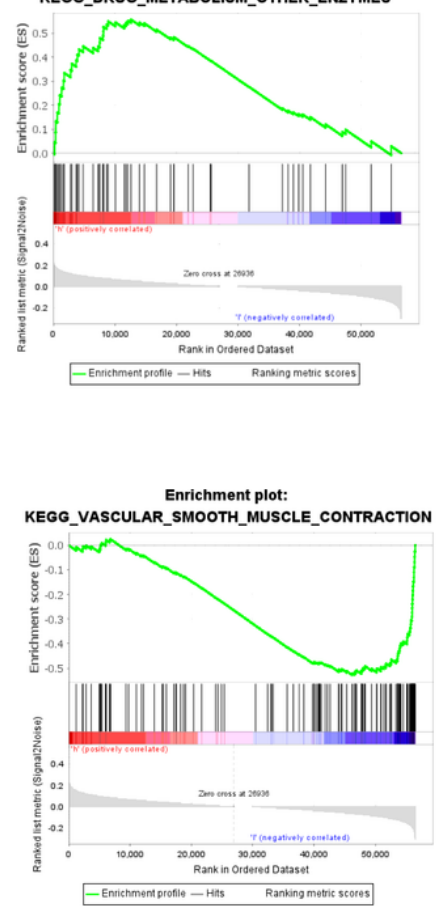

Enrichment plot:

C

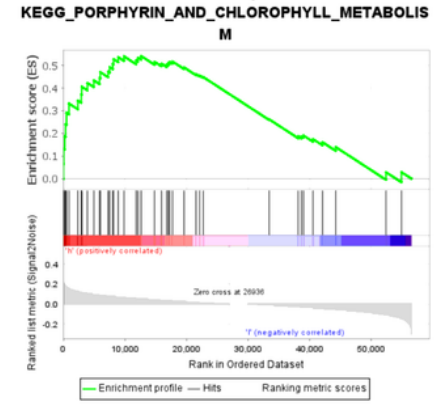

f

Enrichment plot: KEGG_ETHER_LIPID_METABOLISM

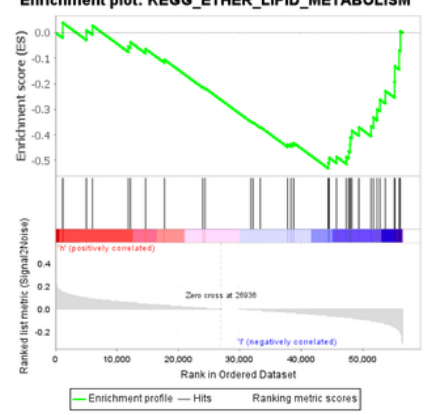

\section{Figure 3}

Gene Set Enrichment Analysis GSEA with high and low TMB groups (a-c) The top3 pathway axis enriched in high TMB groups included proteasome, drug metabolism other enzymes, porphyrin and chlorophyll metabolism. (d-f) The top3 pathway axis enriched in low TMB groups were ECM receptor interaction, vascular smooth muscle contraction, and ether lipid metabolism. 


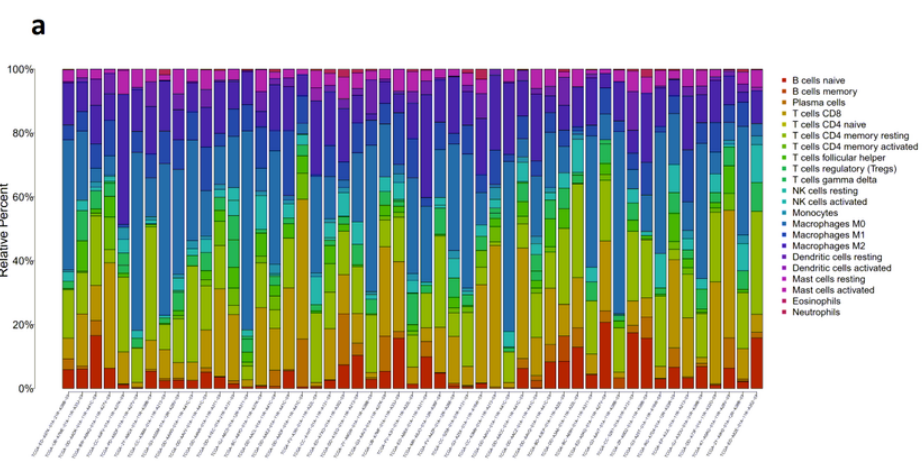

b

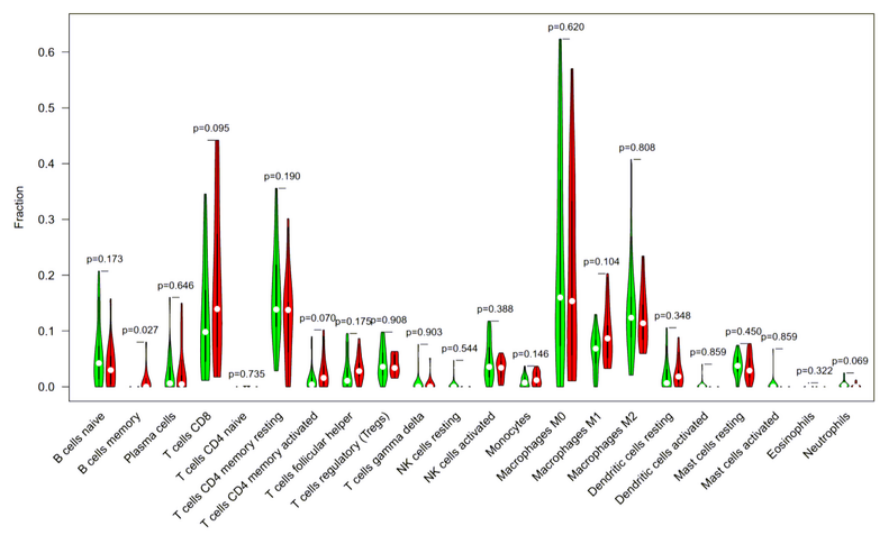

Figure 4

Tumor-infiltrating immune cells in hepatocellular carcinoma (a) The stacked bar graph showed the infiltration of 22 immune cells in each sample. Each color represented a type of immune cell. (b) The Wilcoxon rank-sum test displayed that memory B cells $(P=0.02), C D 8+T$ cells $(P=0.09), C D 4+$ memory activated $T$ cells $(P=0.07)$ and neutrophils $(P=0.06)$ had a difference in high and low TMB groups. 

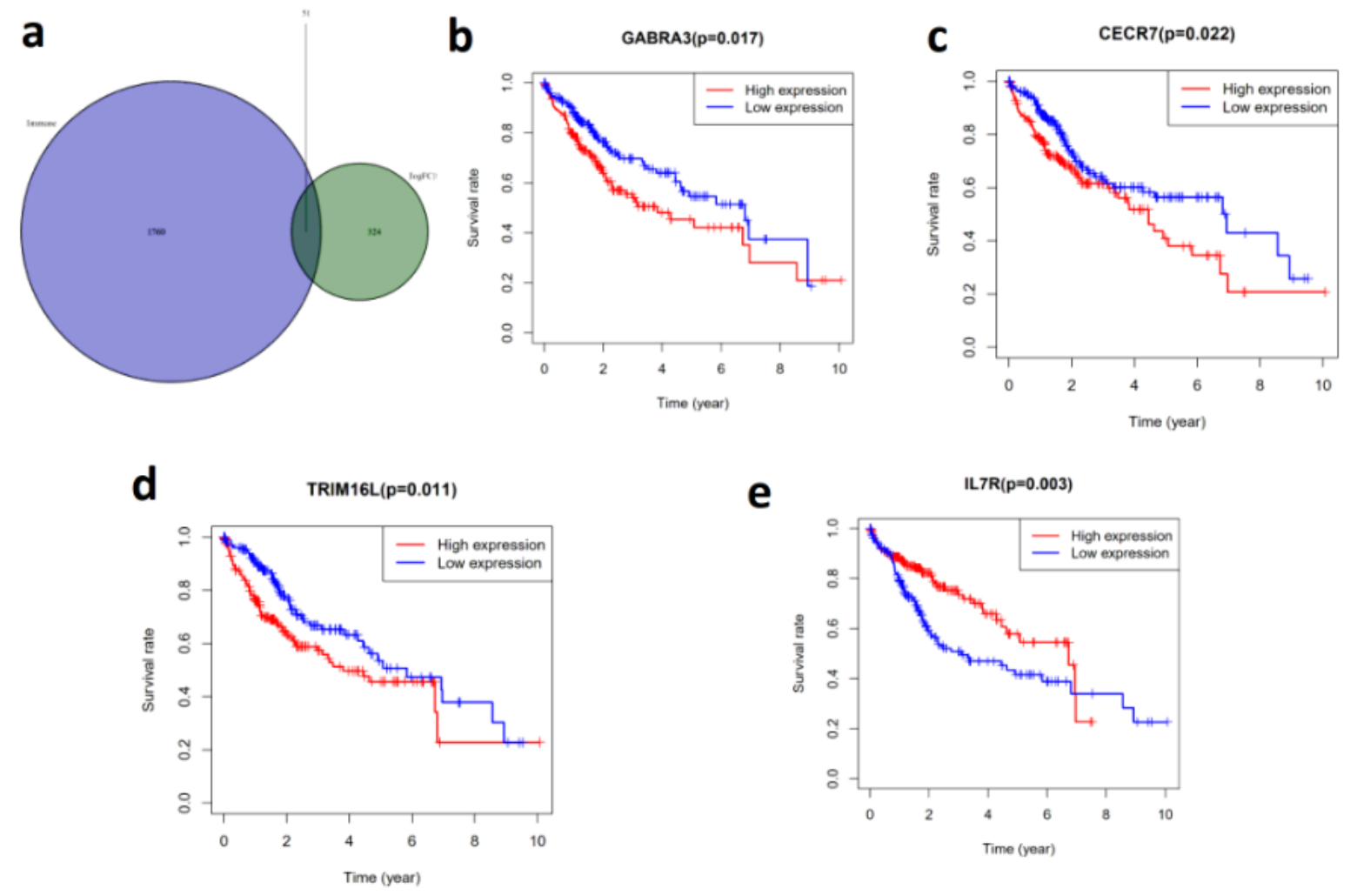

\section{Figure 5}

Identification of significant immune genes for HCC prognostication (a) The Venn diagram showed that a total of 51 differential immune genes were associated with tumor mutation burden and immune infiltration. Kaplan-Meier analysis revealed that down-expression of GABRA3, CECR7, TRIM16 and upexpression of IL7R were associated with better survival outcomes and low recurrence. (b) GABRA3(P=0.0017) (c) CECR7 ( $P=0.022)(d)$ TRIM16L( $P=0.011)(e)$ $\operatorname{IL7R}(\mathrm{P}=0.003)$
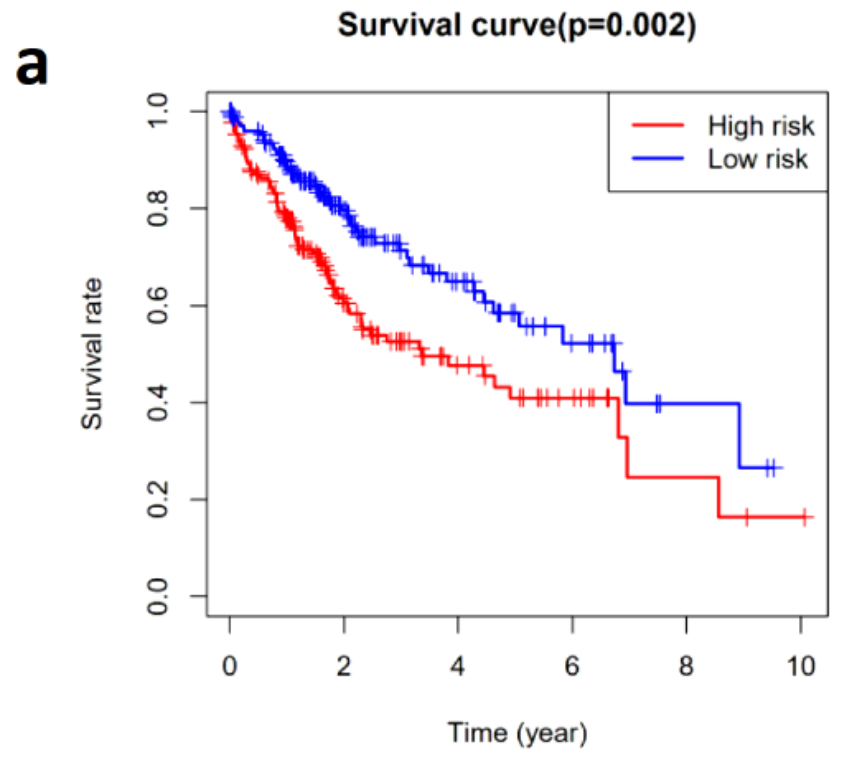

b

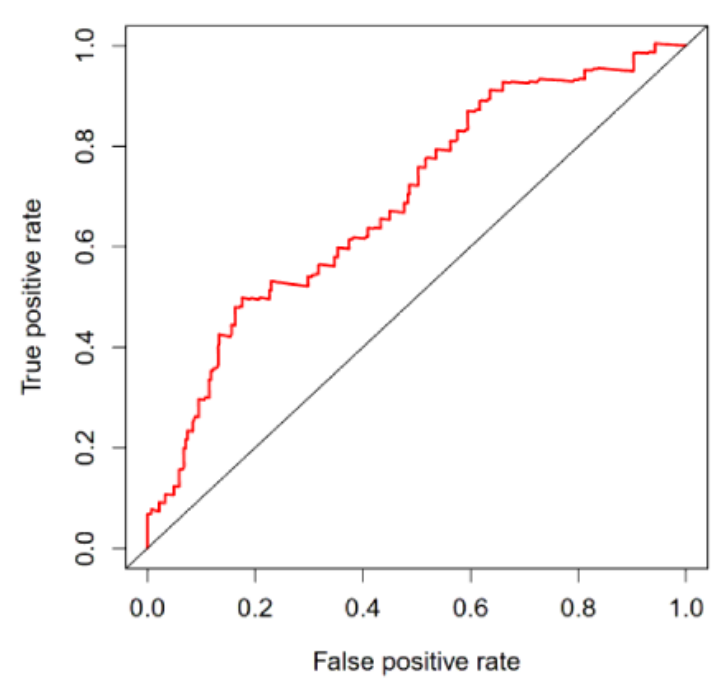

\section{Figure 6}

Analysis and evaluation of the risk prediction (diagnosis) models in $\mathrm{HCC}$ (a) Kaplan-Meier analysis demonstrated that patients with higher risk showed worse survival rate $(p=0.002)$. (b) The AUC of ROC curve $(A U C=0.69)$ showed the predictive accuracy of TMB risk scores. AUC, area under curve; HCC, hepatocellular carcinoma; ROC, receiver operating characteristic. 


\section{Supplementary Files}

This is a list of supplementary files associated with this preprint. Click to download.

- Supplementarymaterials.docx

- Supplementarymaterials.docx

- FigureS3.tif

- Figures3.tif

- Figures2.tif

- Figures2.tif

- FigureS1.tif

- Figures1.tif

- tables2.docx

- tables2.docx

- tableS1.docx

- tables1.docx 